\title{
A NEW PARAMETERIZATION OF POLAR MOTION
}

\author{
Haim B. Papo \\ Department of Civil Engineering \\ Technion, Haifa, Israel
}

\section{ABSTRACT}

The rotational motion of the earth is decomposed into spin,polar motion and local motions. The rotation vector components are associated to phenomena such as precession, nutation, diurnal spin,polar motion and local motions. The above decomposition is accomplished without refering to an earth-fixed CIO pole or BIH zero meridian. The time-like variations of the coordinates of a surface point in a geocentric equatorial reference frame are presented as a function of the rotation vector components. In the rigid earth approximation three scalar parameters are necessary for evaluating point coordinate variations, namely spin rate of the earth, polar motion magnitude and spin rate of the polar motion vector. Two numerical examples are given as an illustration.

\section{INTRODUCTION}

The polar motion phenomena has been continuously studied, measured and analysed for over a century by an ever-increasing number of devotees. The past two decades have been marked by extensive refinements in the dynamical analysis of the rotation of the earth and by the advent of new observational systems of superior accuracy (VLBI, LASER ranging). Grand designs are under way with a promise of polar motion determination with centimeter level accuracy. Somehow, the objectives of all those efforts have not changed. The ultimate results sought are still the age-old $\mathrm{x}, \mathrm{y}$ angles. The purpose of this paper is to challenge the uniqueness of the classic $x, y$ polar motion parameters. It is suggested that rather than the $\mathrm{x}, \mathrm{y}$ angles, their time rates be determined,presented as a vector and related to the diurnal spin vector of the earth.

REFERENCE FRAMES AND THE ROTATION VECTOR

The basic reference frames needed in the present analysis are briefly defined, where a more complete discussion may be seen in Grafarend et.al. (1979). The inertial frame e is defined by a star catalog system, where the $e^{l}, e^{3}$ axes are identified respectively with the mean equinox and

E. M. Gaposchkin and B. Kotaczek (eds.), Reference Coordinate Systems for Earth Dynamics, 119-123. Copyright $\odot 1981$ by D. Reidel Publishing Company. 
pole of the catalog at the standard epoch. The equatorial frame $e_{3}$ is defined by the observable spin axis of the earth (See Atkinson,1975 and Leick, 1978) and the mean ecliptic at epoch $T$. The time-like variations of $e_{3}$ vs. e are modeled by the parameters of precession and forced nutation (without diurnal frequencies). The conventional frame $e_{c}$ defined by a reference pole and zero meridian is explicitly related to a net of stations at a particular epoch. An arbitrary point $p$ on the earth surface is defined by its geocentric position vector $\bar{p}$ which is refered to the $e_{3}$ frame by geocentric colatitude $\sigma$ and hour angle $h$.

The rotation vector $\bar{\omega}$ of a material point $p$ on a deformable earth is defined as the vorticity of the local velocity field (See Grafarend et. al.,1979). This definition is instrumental for partitioning the timelike variations in position of $\mathrm{p}$ into rotations,translations and deformations. The vector $\bar{w}$ representing the complete spectrum of rotational motions of $\mathrm{p}$ vs. a geocentric inertial frame is partitioned into the following components:

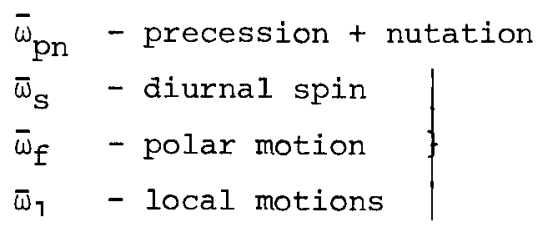

derived from theory

determined from observations

The first three components of $\bar{\omega}$ are global, i.e., space-invariant, same for any $p$ point. At a given epoch they represent the rotation of the earth crust in an average sense. Regarding $\sigma, h$ of a station as the directly observable quantities we are interested in the spin,polar motion and local motions components of the rotation vector.

\section{REPARAMETERIZATION OF POLAR MOTION}

Universal time UT1 or equivalently $\theta$. The apparent sidereal angle and the polar motion parameters $x, y$ are essentially transformation angles through which the conventional reference frame $e_{c}$ is related to $e_{3}$ the equatorial frame at a given epoch. The time-like variations of those angles, denoted respectively by $\dot{\theta}, \dot{x}, \dot{y}$, represent the rotational motion of the conventional vs. the equatorial frames. But these same motions were defined above by the diurnal spin $\bar{\omega}_{S}$ and polar motion $\bar{\omega}_{f}$ rotation vector components. From Figure 1 the functional relationship between the two sets of parameters is derived as follows:

$$
\begin{aligned}
& \left|\bar{\omega}_{s}\right|=\dot{\theta}=\omega_{s} \quad-\text { spin rate of the earth (vs. } e_{3} \text { ) } \\
& \left|\bar{\omega}_{f}\right|=\sqrt{\dot{x}^{2}+\dot{y}^{2}}=\omega_{f} \quad-\text { magnitude of polar motion vector } \\
& h_{f}=\theta-\arctan (\dot{y} / \dot{x})-\Pi / 2 \quad-\text { hour angle of polar motion vector } \\
& \dot{h}_{f}=\dot{\theta}+(\ddot{x} \dot{y}-y \dot{x}) / \omega_{f}^{2}
\end{aligned}
$$

where $\ddot{x}, \ddot{y}$ are the respective second derivatives of $x, y$ vs. time. As the proposed parameters $\omega_{S}, \omega_{f}, \hat{h}_{f}$ are functions of the time derivatives of UTl, $x, y$ they also have irregular time-like variations and have to be determined empirically from observations on a day by day basis. 
Given a discrete series of $\omega_{S}, \omega_{f}, \hat{h}_{f}$ values, UTl, $x, y$ can be evaluated for any desired epoch by quadrature. The set of initial values (UTI, $x, y$ ) at the zero epoch is equivalent to the selection of a particular conventional reference frame (CIO/BIH being only one out of many possibilities) Thus we see that the proposed set of parameters is fundamentally different from the one currently in use by being completely independent of the particular choice of conventional frame.

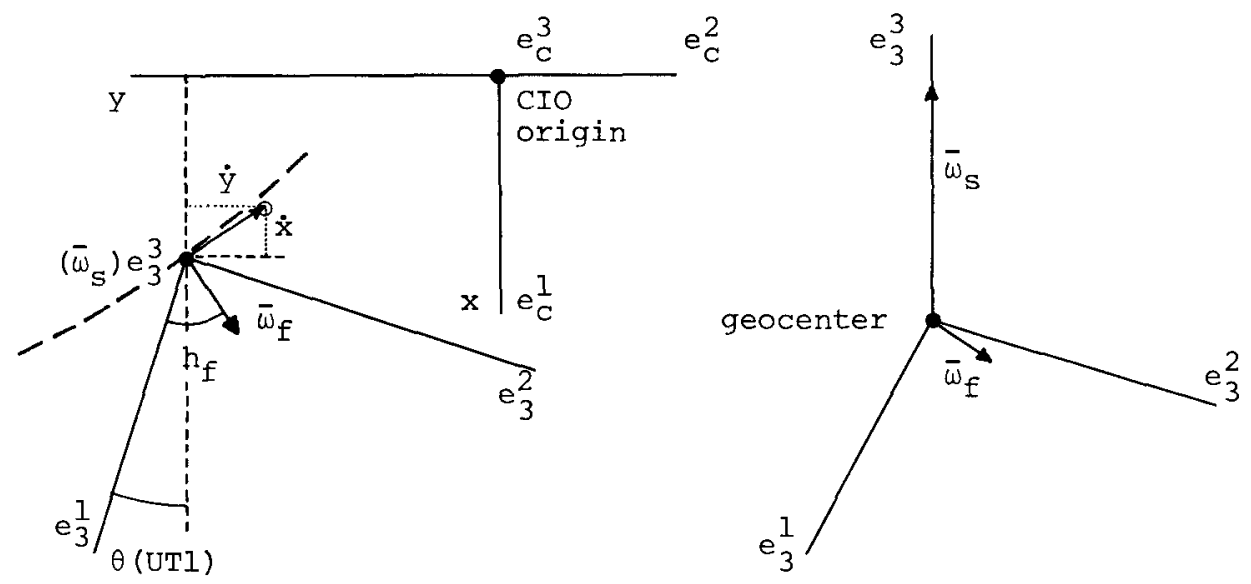

Figure 1. The polar motion vector

A simple numerical exercise was performed to obtain some feeling for the magnitudes of the proposed polar motion parameters. Table 7 in the 1977 BIH Annual Report ( $x, y$, UTl-ATl smoothed values at 5 day intervals) was differentiated numerically and transformed into $\omega_{S}, \omega_{f}, \dot{h}_{f}$. Table 1 lists the results at 40 day intervals.

Table 1. Spin and polar motion parameters for 1977.

\begin{tabular}{rrrr}
\hline $\begin{array}{c}\text { Epoch } \\
\text { day }\end{array}$ & $\begin{array}{c}\omega_{\mathrm{s}^{-2301 .}} \\
\mathrm{rad} / \text { year }\end{array}$ & $\begin{array}{c}\dot{\mathrm{h}}_{\mathrm{f}} \\
\mathrm{rad} / \text { year }\end{array}$ & $\begin{array}{c}\omega_{\mathrm{f}} \\
\mathrm{micrad} / \text { year }\end{array}$ \\
\hline 0 & 0.167305 & 10.7319 & 6.3278 \\
40 & 308 & 4.1028 & 8.0486 \\
80 & 295 & 7.6559 & 6.7288 \\
120 & 293 & 5.5007 & 6.6373 \\
160 & 315 & 3.3389 & 7.9860 \\
200 & 330 & 5.3783 & 8.1570 \\
240 & 314 & 7.1653 & 7.3203 \\
280 & 295 & 8.9587 & 7.1117 \\
320 & 296 & 3.7296 & 6.4164 \\
360 & 292 & 7.7184 & 7.8234 \\
\hline
\end{tabular}

VARIATION OF COORDINATES DUE TO POLAR MOTION

The time-like variation of $\bar{p}$ in $e_{3}$ due to $\bar{\omega}_{S}$ and $\bar{\omega}_{f}$ is given by:

$$
\dot{\mathrm{p}}=\bar{\omega}_{\mathrm{s}} \times \overline{\mathrm{p}}+\bar{\omega}_{\mathrm{f}} \times \overline{\mathrm{p}} \text {. }
$$


From equivalent matrix expressions for the vector product or using spherical trigonometry one can easily derive the following equations:

$$
\begin{aligned}
& \dot{\sigma}=0-\omega_{f} \sin \left(h-h_{f}\right) \\
& \dot{h}=\omega_{s}-\omega_{f} \cos \left(h-h_{f}\right) \cot \sigma
\end{aligned}
$$

The terms in the above expressions due to $\bar{\omega}_{f}$ can be obtained also from Mueller (1969,pp.87) by differentiating equations 4.39 and 4.40 and transforming from $\dot{x}, \dot{y}$ to $\omega_{f}, h_{f}$ variables. In order to demonstrate the utility of the proposed polar motion parameters we evaluated the effect of simulated polar motion on the geocentric equatorial coordinates of five stations. The following constants were taken from Markovitz (1976):

$$
\begin{aligned}
& \omega_{s}=2301.1676 \mathrm{rad} / \text { year } \\
& \dot{\omega}_{f}=3.86309 \mathrm{microrad} / \text { year } \\
& \dot{\mathrm{h}}_{\mathrm{f}}=2306.4797 \mathrm{rad} / \text { year }
\end{aligned}
$$

The period of the polar motion vector $2 \pi /\left(\dot{h}_{f}-\omega_{S}\right)=1.1828$ years is close to Chandler's period. The coordinates of the five stations simulating the ILS network at the initial epoch $\mathrm{T}_{0}$ were:

$$
\begin{aligned}
& \sigma_{1}=\sigma_{2}=\sigma_{3}=\sigma_{4}=\sigma_{5}=50^{\circ} \\
& h_{1}=10^{\circ} ; h_{2}=82^{\circ} ; h_{3}=154^{\circ} ; h_{4}=226^{\circ} ; h_{5}=298^{\circ}
\end{aligned}
$$

The value of $h_{f}$ at $T_{0}$ was set to be $\pi$. Using an ordinary numerical integration procedure $\sigma_{i}, h_{i}$ were evaluated over a period of 440 sidereal days. Table 2 shows the variations in $\sigma_{i}$ and $h_{i}$ at 40 day intervals. In Figure 2 we plotted the varying coordinates of station 1 at the beginning of each sidereal day, using polar stereographic projection with differend scales for $\sigma_{1}$ on one hand and for $\Delta \sigma_{1}, \Delta \mathrm{h}_{1}$ on the other. We plotted also the varying position of a reference pole which is at equal distances from the five stations. The resulting circle with a radius of 0.15 is the inverse of polar motion as it shows the path of the reference pole with respect to the equatorial $e_{3}$ frame.

Table 2. Variations in equatorial coordinates of five stations (0.01)

\begin{tabular}{rrrrrrrrrrr}
\hline Epoch & $\Delta \mathrm{h}_{1}$ & $\Delta \sigma_{1}$ & $\Delta \mathrm{h}_{2}$ & $\Delta \sigma_{2}$ & $\Delta \mathrm{h}_{3}$ & $\Delta \sigma_{3}$ & $\Delta \mathrm{h}_{4}$ & $\Delta \sigma_{4}$ & $\Delta \mathrm{h}_{5}$ & $\Delta \sigma_{5}$ \\
\hline 0 & 0 & 0 & 0 & 0 & 0 & 0 & 0 & 0 & 0 & 0 \\
40 & 7 & -1 & 3 & 8 & -5 & 6 & -6 & -4 & 1 & -8 \\
80 & 13 & -6 & 9 & 12 & -7 & 14 & -13 & -4 & -1 & -16 \\
120 & 15 & -15 & 16 & 12 & -5 & 22 & -19 & 2 & -7 & -21 \\
160 & 13 & -23 & 22 & 7 & 1 & 27 & -22 & 10 & -14 & -22 \\
200 & 7 & -28 & 25 & -1 & 8 & 28 & -20 & 18 & -20 & -17 \\
240 & 0 & -30 & 24 & -9 & 15 & 24 & -15 & 24 & -23 & -9 \\
280 & -6 & -26 & 19 & -15 & 18 & 16 & -7 & 25 & -22 & -1 \\
320 & -10 & -18 & 12 & -17 & 17 & 8 & -1 & 22 & -18 & 6 \\
360 & -10 & -10 & 5 & -14 & 13 & 1 & 3 & 15 & -11 & 8 \\
400 & -5 & -3 & 1 & -7 & 6 & -2 & 3 & 6 & -4 & 5 \\
440 & 1 & 0 & 0 & 1 & -1 & 1 & -1 & -1 & 1 & -1 \\
\hline
\end{tabular}




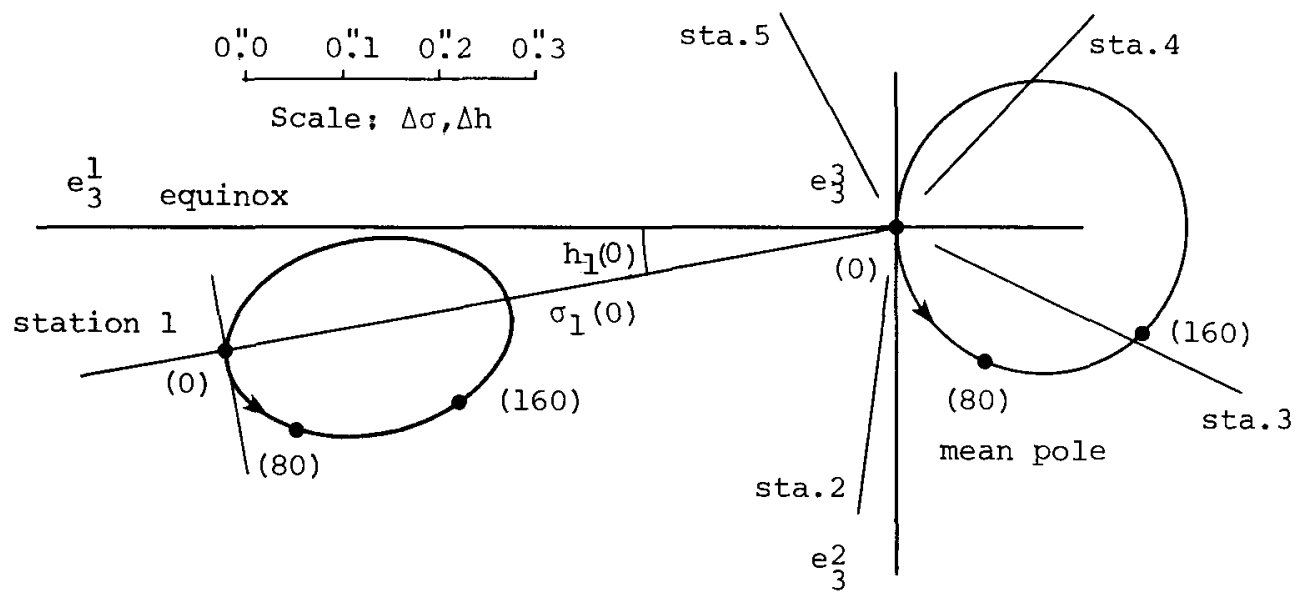

Figure 2. Variation of coordinates due to polar motion.

\section{SUMMARY}

The proposed reparameterization of polar motion is not just another mathematical gag. It should be seriously considered for analysis and representation of earth rotation. Its primary merit is in avoiding any dependence on a fixed conventional origin (pole) and zero meridian. At any time and by an explicit formulation a conventional reference frame may be derived from the varying positions of a network of monitoring stations. This seems the right way of analysing modern, high accuracy observations without blurring their accuracy by artifacts which are in open contradiction to the behaviour of the real earth.

\section{ACKNOWLEDGMENT}

Most of the study reported in the above paper was carried out while the author was on sabbatical leave with Prof. I.I. Mueller at OSU. Working with Prof. Mueller was exciting, challenging and highly rewarding.Partial support for the study was provided by NASA/GSFC Grant No. NSG 5265.

\section{REFERENCES}

Atkinson R.d'E. :1975,"On theEarth's Axes of Rotation and Figure" Mon. Not.R.Astr.Soc. ,71,pp.381-386.

Grafarend E.W.,Mueller I.I.,Papo H.B., Richter B. :1979,"Concepts for Reference Frames in Geodesy and Geodynamics",Bull.Geod. ,53,No.3.

Leick A. :1978, "The Observability of the Celestial Pole and its Nutations" OSU Dept. of Gedetic Science, Report No.262, Columbus.

Markovitz W. :1976,"Comparizon of ILS,IPMS,BIH and Doppler Polar Motion with Theoretical", Report to IAU Comm. 19 and 31,XVI General Assembly, Grenoble, France.

Mueller I.I. :1969,"Spherical and Practical Astronomy as Applied to Geodesy",Frederick Ungar Publ.Co. ,New York. 